UNUSUAL SHADOWING EFFECTS IN PARTICLE PRODUCTION OFF NUCLEI*

R. Blankenbecler

Stanford Linear Accelerator Center Stanford University, Stanford, California 94305

A. Cape11a, J. Tran Thanh Van

Laboratoire de Physique Theorique et Hautes Energies University of Paris XI

Orsay, France 91405

and

C. Pajares, A. V. Ramallo

Departmento de Fisica Teorica

Universidad de Santiago de Compostela, Spain

\begin{abstract}
Some consequences of the AKG cutting rules for particle production off nuclei at low transverse momentum are examined. The condition for an arbitrarily defined cross section to be shadowed only by itself is given. Some physical examples and experimental consequences are discussed. Many new tests of certain general features of Reggeon field theory follow from our results.
\end{abstract}

Submitted to Physics Letters B

* Work supported by the Department of Energy, contract DE-AC03-76SF00515. 
The cutting rules in Reggeon Field Theory [1] have a very general validity; they follow from analiticity, unitarity and large $\mathrm{p}_{\mathrm{T}}$ damping [2]. The purpose of this letter is to explore in a systematic way certain consequences of these rules in hadron-nucleus interactions. Some of the formulae below can be found scattered thrughout the 1iterature, mostly in the context of one and two particle inclusive reactions. However, it does not seem to be recognized that these rules have a much broader domain of applicability. Indeed, they have farreaching consequences in many physical situations in which adequate selection criteria are imposed on the physical events.

The resulting formulae apply with trivial modifications to both hadron-hadron and hadron-nucleus interactions. We restrict our analysis to the latter becausem, in this case, some of the consequences of the cutting rules (such as absence of shadowing or even presence of antishadowing) are most dramatic. For simplicity we shall use the implementation of the cutting rules contained in a simple probabilistic version of the Glauber-Gribov model [4]. However, our main results are independent of this model. Testing these results provides then a model independent test of the basic assumptions of the Reggeon Field Theory. Some of these tests are already possible with available data and are presented below. Other tests are suggested.

Consider the scattering hA of a hadron $h$ on a nucleus of atomic number A. The cross section for the scattering of $h$ on $n$ nucleons of the nucleus (with $A-n$, nucleons being spectators) is given by $(n \geqslant 1)$

$$
\sigma_{n}^{h A}(b)=\left(\begin{array}{l}
A \\
n
\end{array}\right)\left[\sigma_{\text {tot }} T(b)\right]^{n}\left[1-\sigma_{\text {tot }} T(B)\right]^{A-n}
$$




$$
\sigma_{n}^{h A}(s)=\int d^{2} b \sigma_{n}^{h A}(b)
$$

In these formulae $\left(\begin{array}{l}A \\ n\end{array}\right)=A ! / n !(A-n) !, \quad \sigma_{\text {tot }}$ is the total h-nucleon cross section, $b$ is the impact parameter, and $T(b)$ is the normalized nuclear hadronic matter distribution. Equation (1) has a straightforward probabilistic interpretation (Bernouilli's formula). Indeed $\sigma_{\text {tot }} \mathrm{T}(\mathrm{b})$ is the probability for the hadron $\mathrm{h}$ to interact with a nucleon from the nucleus at impact parameter b.

\section{Event Selection Criteria}

Single Criteria

Let us now examine the effect of introducing partial cross sections by defining some criterion $\mathrm{C}$, such that only events that satisfy the requirements of $\mathrm{C}$ will be counted; the resulting cross section is $\sigma_{\mathrm{C}}$. Clearly, $\sigma_{\text {tot }}=\sigma_{\mathrm{C}}+\sigma_{\mathrm{N}}$, where $\mu_{\mathrm{N}}$ denotes the cross section corresponding to all events which do not satisfy the requirements of $C$. Now

$$
\left[\mathrm{T}(\mathrm{b}) \sigma_{\text {tot }}\right]^{\mathrm{n}}=\mathrm{T}^{\mathrm{n}}(\mathrm{b}) \sum_{i=0}^{\mathrm{n}}\left(\begin{array}{c}
\mathrm{n} \\
i
\end{array}\right) \sigma_{C}^{i} \sigma_{N}^{\mathrm{n}-i} .
$$

Suppose now that the criterion $C$ is such that a term in eq. (4) is counted if and only if $i \geqslant 1$. Physically this condition means that the superpositions of any number of events satisfying $C$ as well as their superpositions with any number of events not satisfying $C$, also do satisfy criterion $C$. Moreover, these are the only events satisfying C. The corresponding cross-section in h-A interaction is then given by 


$$
\begin{aligned}
{ }_{\sigma_{C}}^{h A}(b) & =\sum_{n=1}^{A}\left(\begin{array}{c}
A \\
n
\end{array}\right)\left[\sigma_{t o t}^{n}-\sigma_{N}^{n}\right] T^{n}(b)\left[1-T(b) \sigma_{\text {tot }}\right]^{A-n} \\
& =1-\left[1-T(b) \sigma_{C}\right]^{A} .
\end{aligned}
$$

Note that in starting the summation in eq. (4) at $n=1$, we implicitly assume that all events satisfying criterion $\mathrm{C}$ are inelastic. Equation (4) has a very interesting consequence. Indeed we see that $\sigma_{\text {tot }}$ (or $\sigma_{\mathrm{N}}$ ) does not appear in the formula and all the absorptive (shadowing) corrections to $\sigma_{C}$ are $O\left(\sigma_{C}^{2}\right)$. If $\sigma_{C} \approx \sigma_{\text {tot }}$ one gets the standard $A^{2 / 3}$ behavior. On the other hand, if $\sigma_{C} \ll \sigma_{\text {tot }}$ one gets $\sigma_{C}{ }_{C} \simeq A \sigma_{C} \cdot$

The main feature of eq. (4), namely that $\sigma_{C}$ is only shadowed by itself, is independent of the elastic rescattering approximation.

The linearity in $A$ should be true in a range of $A$ such that $\sigma_{C}(A-1) \ll 2 \pi R^{2}(A)$, where the effective nuclear radius $R(A)$, is defined by $\int d^{2} b[t(b)]^{2}=1 /\left[\pi R^{2}(A)\right]$. However, the particular form of the self-shadowing given by eq. (4) does depend on the elastic rescattering approximation. A derivation of this result using only the A.K.G. cutting rules can be found in the Appendix of ref. [4]

Examples:

I. Define $\mathrm{C}$ to be all inelastic diffractive events. Thus $\sigma_{\mathrm{C}}=\sigma_{\text {in }}$ and $\sigma_{N}=\sigma_{e l}$. It is easy to see that this is a suitable criterion: the superposition of any number of inelastic events as well as their superposition with elastic events is an inelastic event. Thus one has the 
well-known result

$\therefore$

$$
\sigma_{\text {in }}^{\mathrm{hA}}(\mathrm{b})=1-\left[1-\mathrm{T}(\mathrm{b}) \sigma_{\text {in }}\right]^{\mathrm{A}} \text {. }
$$

This equation implies that the hadron-nucleon inelastic cross-section is shadowed by itself [3] and not by $\sigma_{\text {tot }}$, and since $\sigma_{\text {in }}$ is large, $\sigma_{\text {in }}^{\text {hA }}$ behaves essentially as $\mathrm{A}^{2 / 3}$.

II. Define $C$ to be all events in which one or more particles of a given type M (for instance positively charged particles) are produced in the rapidity interval $\mathrm{y}_{1}<\mathrm{y}<\mathrm{y}_{2}$. It is easy to see that this is a suitable criterion and thus eq. (4) applies to this case. If $\Delta=y_{2}-y_{1}$ is small, $\sigma_{C}$ is small, and $\sigma_{C}^{h A}$ will have a linear $A$ dependence. By increasing $\Delta, \sigma_{\mathrm{C}}$ will be increased and $\sigma_{\mathrm{C}}^{\mathrm{hA}}$ can eventually behave as $\mathrm{A}^{2 / 3}$. It would be interesting to check experimenta1ly this dependence on $\Delta$ of the A-dependence, parametrized, for instance, as $\sigma_{C}^{h A} \propto A^{\alpha(\Delta)}$. Note that if $C$ is defined to be one and only one particle of type $M$ within $\Delta$, this does not satisfy the test leading to eq. (4).

A physically relevant example is to choose $M$ to be a $J / \psi$ particle produced inside $\Delta$. Since $\sigma_{\mathrm{J} / \psi}$ is small for all values of $\Delta$, one finds $\alpha(\Delta)=1$

III. A most interesting example is $\overrightarrow{p A}$ reactions [5]. Define $c$ to be annihilation events, i.e., events with no fast isolated antibaryons $(B \bar{B}$ pair production is allowed for fast pairs but the corresponding 
cross section is negligibly smal1). Then $\sigma_{C}=\sigma_{\text {annih, }}$, and since this cross section is small on a proton target at high energies, one expects $\sigma_{\text {annih }} \propto \mathrm{A}$. A similar example can be found in $\mathrm{pA}$ reactions in which $\mathrm{C}$ is defined as the set of events with no fast isolated baryons. This forces $\sigma_{C}$ to be small at high energies since a baryon Regge exchange is involved and one expects $\sigma_{\mathrm{C}}^{\mathrm{pA}} \propto \mathrm{A}$. We show below that this behavior is in agreement with experiment.

IV. If in example II one takes $y=y+\delta y$ with $\delta y \operatorname{sma} 11, \sigma_{C}$ is related to the inclusive cross section: $\sigma_{C}=(d \sigma / d y) \delta y$. Using eq. (4) and taking the limit $\delta y \rightarrow 0$ one gets the well known result [1]

$$
\frac{d \sigma}{d y}(h A \rightarrow M X, y)=A \frac{d \sigma}{d y}(h p \rightarrow M X, y)
$$

Obviously, such a relation is then also true for $E d^{3} \sigma / d^{3} p$.

Double Criteria

Let us consider next the partial cross section $\sigma_{\mathrm{CD}}$ corresponding to a11 the events satisfying two different criteria C and D. Obviously

$$
\sigma_{\text {tot }}=\sigma_{C D}+\sigma_{C}+\sigma_{D}+\sigma_{N}
$$

where $\sigma_{C}\left(\sigma_{D}\right)$ is the partial cross-section for all events satisfying criterion $C(D)$ only, and $\sigma_{N}$ the cross section corresponding to all the events that satisfy neither $C$ or $D$. Thus $\left(\sigma_{\text {tot }}\right)^{n}$ is a superposition of 
terms of the form $(i+j+h \leqslant n)$

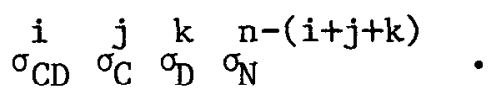

Suppose now that criteria C and D are such that terms of type (8) are counted if and only if $i \geqslant 1$ and/or both $j \geqslant 1$ and $k \geqslant 1$. We can then write

$$
\begin{aligned}
\sigma_{C D}(b) & =\sum_{n=1}^{A}\left(\begin{array}{l}
A \\
n
\end{array}\right)\left[\sigma_{\text {tot }}^{n}-\left(\sigma_{C}+\sigma_{N}\right)^{n}-\left(\sigma_{D}+\sigma_{N}\right)^{n}+\sigma_{N}^{n}\right] \\
& \times[T(b)]^{n}\left[1-T(b) \sigma_{\text {tot }}\right]^{A-n}
\end{aligned}
$$

In eq. (9), all contributions to $\sigma_{\text {tot }}$ not satisfying both criteria $C$ and D have been subtracted out. Performing the summation over $\mathrm{n}$ one gets

$$
\begin{aligned}
\sigma_{C D}^{h A}(b)= & 1-\left[1-T(b)\left(\sigma_{C D}+\sigma_{D}\right)\right]^{A}+1-\left[1-T(b)\left(\sigma_{C D}+\sigma_{D}\right)\right]^{A} \\
& -\left\{1-\left[1-T(b)\left(\sigma_{C D}+\sigma_{C}+\sigma_{D}\right)\right]\right\}
\end{aligned}
$$

The physical interpretation of this equation is quite transparent. Referring to eq. (4) one can see that the first (second) term is the partial cross-section for events satisfying $C(D)$ with or without $D(C)$. The third term is the cross section for events satisfying $C$ or $D$ only, together with those events satisfying both $C$ and $D$ which had been counted in both the first and second term (and thus were double counted). 
We now examine some interesting limits of eq. (10). If $\sigma_{\mathrm{CD}}$ is large one expects an $\mathrm{A}^{2 / 3}$ behavior of $\sigma_{\mathrm{CD}}^{\mathrm{hA}}$. If $\sigma_{\mathrm{CD}}$ and $\sigma_{\mathrm{D}}$ are small and $\sigma_{C}$ is large, one gets

$$
\sigma_{C D}^{h A}(b) \approx A T(b) \sigma_{C D}+A T(b) \sigma_{D}\left[1-\left(1-T(b) \sigma_{C}\right)^{A-1}\right] \text {. }
$$

Since the bracket in the second term is proportional to $\theta(R-b)$ one gets an essentially linear A behavior. Finally if $\sigma_{C D}, \sigma_{C}$ and $\sigma_{D}$ are all sma11, one gets

$$
\sigma_{C D}^{h A}(b) \approx A T(b) \sigma_{C D}+A(A-1) \sigma_{C} \sigma_{D} T^{2}(b)+\ldots
$$

Upon integration over $\mathrm{b}$ one gets here a term in $\mathrm{A} \sigma_{\mathrm{CD}}$ and a term $\sigma_{C} \sigma_{D} A(A-1) /\left[\pi R^{2}\right] \propto A^{4 / 3}$. The effectiver behavior of $\sigma_{C D}^{h A}(s)$ is thus $A^{\alpha}$ with $1<\alpha<4 / 3$, the exact value of $\alpha$ depending on the relative size of the two terms in eq. (12).

\section{Examp1es:}

v. Define $C(D)$ to be all events in which at least one particle of type $M(N)$ is produced in the rapidity interval $y_{1}<y_{M}<y_{2}\left(y_{3}<y_{N} y_{4}\right)$. For instance $\mathrm{M}$ could be a positive and $\mathrm{N}$ a negative charged particle. It is easy to see that these criteria satisfy the above requirements and thus eqs. (10-12) will be valid depending on the values of $\sigma_{\mathrm{CD}}, \sigma_{\mathrm{C}}$ and $\sigma_{D}$. These, in turn, depend on the nature of the produced particle and the size of the rapidity interval in which they have to be produced. 
VI. A physically interesting example is the case where a $\mathrm{J} / \psi$ particle has to be produced between $\mathrm{y}_{1}$ and $\mathrm{y}_{2}$ (criterion $\mathrm{C}$ ) together with at least one particle of type $\mathrm{N}$ between $\mathrm{y}_{3}$ and $\mathrm{y}_{4}$ (criterion $\mathrm{D}$ ). In this situation $\sigma_{C D}$ and $\sigma_{C}$ are small but $\sigma_{D}$ is large if $y_{3}$ and $y_{4}$ are far apart. In this case eq. (10) holds and one gets a linear A dependence. By reducing the rapidity gap $\Delta=y_{4}-y_{3}, \sigma_{D}$ becomes sma11 and eq. (12) applies. If the second term in the latter equation is sizeable, one will get an $\mathrm{A}^{\alpha}$ dependence with $\alpha>1$.

VII. If in example I, one takes $y=y+\delta y$ and $y^{\prime}=y^{\prime}+\delta y^{\prime}$ with $\delta y$ and $\delta y^{\prime}$ sma11, one gets from eq. (12), in the limit $\delta y \rightarrow 0$ and $\delta y^{\prime} \rightarrow 0$, the well known formula for the two paticle inclusive cross section,

$$
\begin{gathered}
\frac{d^{2} \sigma}{d y d y^{\prime}}(h A \rightarrow M+N+X)=A \frac{d^{2} \sigma}{d y d y^{\prime}}(h p \rightarrow M+N+X) \\
+\frac{A(A-1)}{\pi R^{2}} \frac{d \sigma}{d y}(h p \rightarrow M+X) \frac{d \sigma}{d y^{\prime}}(h p \rightarrow N+X) .
\end{gathered}
$$

VIII. Consider a single particle inclusive cross section but with an extra requirement. Suppose now that $C$ is any suitable criteria of the type discussed above and $D$ is again at least one particle of type $M$ in a rapidity interval $\mathrm{y}_{1}<\mathrm{y}<\mathrm{y}_{2}$. Taking $\mathrm{y}=\mathrm{y}+\delta \mathrm{y}$ and going to the limit $\delta y \rightarrow 0$, one gets the partial single particle inclusive cross section in which one counts only the events satisfying criterion $c$. In this case $\sigma_{\mathrm{CD}}=\left(\mathrm{d} \sigma_{\mathrm{C}} / \mathrm{dy}\right) \delta \mathrm{y}, \sigma_{\mathrm{D}}=\left(\mathrm{d} \sigma_{\mathrm{N}} / \mathrm{dy}\right) \delta \mathrm{y}$, and one gets from eq. (11) 


$$
\begin{aligned}
& \stackrel{-d \sigma_{C}}{d y}(h A+M+X)=A \frac{{ }^{d} \sigma_{C}}{d y}(h p \rightarrow M+x) \\
& +A \frac{d \sigma_{N}}{d y}(h p \rightarrow M+X) \int d^{2} b T(b)\left[1-\left(1-T(b) \sigma_{C}\right)^{A-1}\right] .
\end{aligned}
$$

Obviously

$$
\frac{d{ }^{d} C}{d y}(h p \rightarrow M+X)+\frac{d \sigma_{N}}{d y}(h p \rightarrow M+X)=\frac{d \sigma}{d y}(h p \rightarrow M+X) .
$$

Note that eq. (14) is formally identical to eq. (11). It follows from eqs. (9) and (14) that when a criterion of the type discussed above is imposed in the single particle inclusive cross section, $d \sigma_{C}^{h A} / d y$ contains two terms. When $\sigma_{\mathrm{C}}$ is large the second term behaves linearly in $\mathrm{A}$ as the first one. However when $\sigma_{C}$ is small this second term behaves like $A^{4 / 3}$. Below we compute this term in some cases and found it sizeable enough to be detected experimentally [6]. As eqs. (6), (13) and (14) are valid for the invariant cross sections, before integrating over $\mathrm{p}_{\mathrm{T}}$.

\section{Phenomenological Applications}

In order to test the linearity in $A$ of a partial cross section $\sigma_{C}$ with a single criterion [eq. (4)] we consider the second example in III namely proton-nucleus scattering with no fast baryon (say with $\left.x \geqslant x_{0}\right)$. Since the average baryon $B$ multiplicity in the projectile hemisphere is equal to one, we can write 


$$
\sigma_{i n}^{p A} \approx \int_{0}^{\mathrm{x}} \frac{\mathrm{d} \sigma}{\mathrm{dx}}(\mathrm{pA}+\mathrm{B}+\mathrm{X}) \mathrm{dx}+\int_{\mathrm{x}_{0}}^{1} \frac{\mathrm{d} \sigma}{\mathrm{dx}}(\mathrm{pA} \rightarrow \mathrm{B}+\mathrm{X}) \mathrm{dx}
$$

The first term in the r.h.s. of eq. (15), which represents the protonnucleus cross section with no fast baryons, is given by eq. (4) and grows almost linearly with $A$. Since the inelast1c (non-diffractive) cross section $\sigma_{\text {in }}^{\mathrm{pA}}$ is known to grow approximately as $\mathrm{A}^{2 / 3}$, eq. (15) implies that the second term has to grow like $A^{\alpha}$ with $\alpha<2 / 3$. This is the well known phenomenon of attenuation of fast secondaries which has been verified experimentally [7]. If the experimental data on attenuation were precise enough for all produced baryons, one could use them,-together with the experimental values of $\sigma_{i n}^{\mathrm{pA}}$, in order to test the A-behavior of the first term in the r.h.s. of eq. (15).. Since this is not the case we are going to use eq. (15) in a different way: we assume that the A-dependence of its first term is given by eq. (4) and we use the experimental values of $\sigma_{i n}^{\mathrm{pA}}$ in order to determine the A-dependence of the last term in eq. (15). The resulting A-dependence is then compared with the experimental data on attenuation. In order to find the value of $\sigma_{C}$ in eq. (4), we use the inclusive data [8] for pp $\rightarrow B+X$. For $\mathrm{x}_{0}=0.3$ we get $7.5 \pm 0.5 \mathrm{mb}$. The values of the last term with $x_{0}=0.3$ for different values of $A$ are given in table $I$. If parametrized in the form $A^{\alpha}$, we find for the value of $\alpha$ between carbon and lead, $\alpha=0.53 \pm 0.01$. The comparison with experimental data is shown in fig. 1. Taking in eq. (15), $x_{0}<0.3\left(x_{0}>0.3\right)$, the value of $\alpha$ increases (decreases) monotonically. Such an $x$-dependence is clearly seen in the data. This indirect test of eq. (4) is quite satisfactory. 
Finally we examine the numerical values of the last term in eq. (14) in the case where $M$ is any charged particle and $\sigma_{C}$ is the annihilation $\overline{\mathrm{p}}-\mathrm{p}$ cross section. Using $\sigma_{C}=\sigma_{\text {annih }}^{\bar{p} p}=7 \mathrm{mb}$, we compute the values of the coefficient of the last term in eq. (14) (table II). We see that this term is quite sizeable at large A. As an application, we can compute the average value, $\left\langle\mathrm{p}_{\mathrm{T}}^{2}\right\rangle_{\mathrm{p} A}^{\overline{\mathrm{p}}}$ annih of the transverse momentum squared in antiproton-nucleus annihilation events. Using eq. (14) and table II, we get:

$$
\begin{aligned}
& \left\langle\mathrm{p}_{\mathrm{T}}^{2}\right\rangle_{\text {annih }}^{\overline{\mathrm{p}} \mathrm{c}}=12\left[\left\langle\mathrm{p}_{\mathrm{T}}^{2}\right\rangle_{\mathrm{a}}^{\overline{\mathrm{p}}}+0.19\left\langle\mathrm{p}_{\mathrm{T}}^{2}\right\rangle_{\text {na }}^{\overline{\mathrm{p}}}\right] \\
& -\left\langle\mathrm{p}_{\mathrm{T}}^{2}\right\rangle_{\text {annih }}^{\overline{\mathrm{p} p}}=207\left[\left\langle\mathrm{p}_{\mathrm{T}}^{2}\right\rangle^{\overline{\mathrm{pp}}}+0.60\left\langle\mathrm{p}_{\mathrm{T}}^{2}\right\rangle_{\text {na }}^{\overline{\mathrm{p} p}}\right] .
\end{aligned}
$$

Such a departure from a linear A-dependence is quite sizeable and should be detectable. Experimentally $\left\langle\mathrm{p}_{\mathrm{T}}\right\rangle_{\mathrm{a}}$ seems to be slightly larger than $\left\langle\mathrm{p}_{\mathrm{T}}\right\rangle_{\mathrm{na}}[9]$. This is expected from the above results. Indeed, due to the small shadowing in annihilation as compared to non-annihilation amplitudes, we expect the latter to be more peripheral that the former, both in $\bar{p} \mathrm{p}$ and $\overline{\mathrm{p}} \mathrm{A}$ interactions.

It is a pleasure to thank A. Krzywicki and J. Kwiecinski for many enlightening discussions.

One of us (R.B.) wishes to thank the LPTHE at the Universite of Paris-Sud, ORSAY, for financial support and kind hospitality while this work was carried out. 
REFERENCES

[1] V. A. Abramovski, 0. V. Kancheli and V. N. Gribov, Proceedings of the 16th International Conference on High Energy Physics, Chicago, Batavia, 1972 .

[2] A. Capella, and A. Krzywicki, Phys. Rev. D18 (1978) 3357.

[3] This result is derived in a different way in ref. [2].

[4] A. Capella, J. Kaplan and J. Tran Thanh Van, Nucl. Phys. B105 (1976) 333.

[5] C. Pajares et a1., to be published.

[6] -For massive lepton-pair production, one finds both a term linear in $\Lambda$ and one that behaves as $A^{4 / 3}$. The coefficient of the latter term falls faster than the first as the pair mass increases. It would be interesting to analyze the data to yield $\alpha$ as a function of the pair mass to see if it overshoots unity at lower masses.

[7] P. Skubic et a1., Phys. Rev. D18 (1978) 3115; A. E. Brenner et a1., Communication at the Madison Conference, Wisconsin, July 1980.

[8] Estimation of the proton-proton cross section with no fast baryon is made from fig. 16 of B. Andersson, Proceedings of the XIth Symposium on Multiparticle Dynamics (1980), p. 422.

[9] D. Everett et a1., Nucl. Phys. B73 (1974) 440; D. R. Ward V, N⿳亠丷⿵冂丶 Symposium Bressanone (1980), p. 459 . 
TABLE I

Proton-proton cross sections with fast baryon

\begin{tabular}{cc}
\hline A & $\sigma_{N}=\sigma_{\text {in }}^{\mathrm{pA}}-\sigma_{\mathrm{C}}^{\mathrm{pA}}$ \\
& \\
\hline 12 & 158,5 \\
27 & 263,4 \\
63 & 418,6 \\
108 & 543,3 \\
207 & 719,8 \\
\hline
\end{tabular}


TABLE II

$$
\begin{gathered}
I=\int d^{2} b \text { T(b) }\left[1-\left(1-T(b) \sigma_{a}\right)^{A-1}\right] \\
\text { with } \sigma_{a}=7 \mathrm{mb}
\end{gathered}
$$

\begin{tabular}{cc}
\hline A & I \\
\hline 12 & 0.19 \\
27 & 0.30 \\
63 & 0.42 \\
108 & 0.50 \\
207 & 0.60 \\
\hline
\end{tabular}


FIGURE CAPTIONS

Fig. 1. The A-dependence of the $p A \rightarrow p(\Lambda)+X$ inclusive spectrum in the proton fragmentation region, parametrized in the form $A^{\alpha}$. The data are from ref. [7]. The dashed surface is the average value of $\alpha$ in the range $x_{0}<x<1$ with $x_{0}=0.3$ computed from eq. (15). Increasing (decreasing) the value of $x_{O}$, one obtains a smaller (larger) value of $\alpha$, in agreement with the data. 


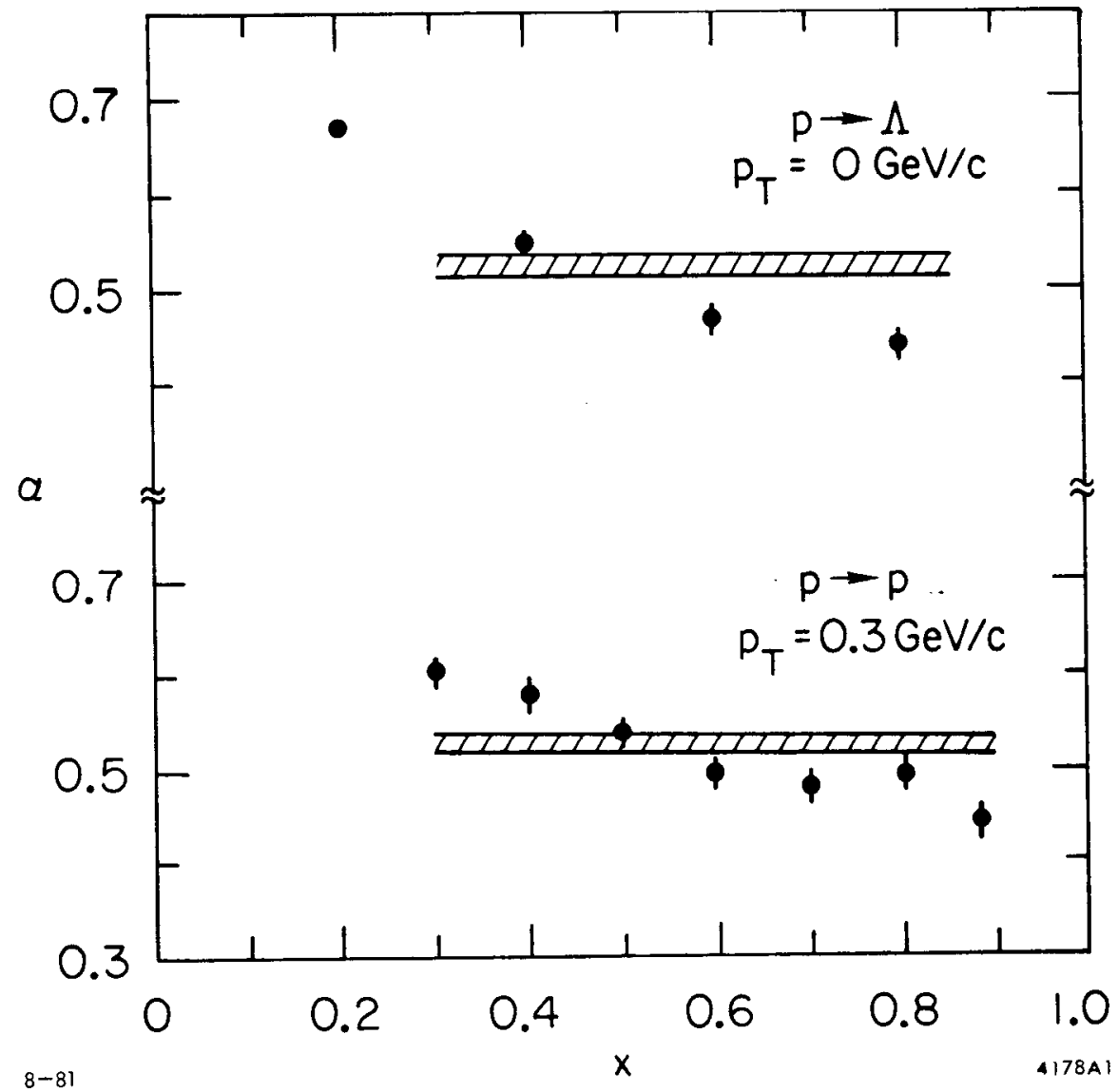

Fig. 1 